\title{
Comparison of two models for phonon assisted tunneling field enhanced emission from defects in Ge measured by DLTS
}

\author{
J.Pienaar*, W.E. Meyer, F. D. Auret and S.M.M. Coelho \\ Department of Physics, University of Pretoria, Pretoria, 0002, South Africa \\ Corresponding author e-mail address: jac pienaar@hotmail.com \\ Tel: 0124202668 \\ Fax: 0126541110
}

\section{Abstract}

Deep Level Transient Spectroscopy (DLTS) was used to measure the field enhanced emission rate from a defect introduced in $n$-type Ge. The defect was introduced through low energy $( \pm 80 \mathrm{eV})$ inductively coupled plasma (ICP) etching using Ar. The defect, named $\mathrm{EP}_{0.31}$ had an energy level $0.31 \mathrm{eV}$ below the conduction band. Models of Pons \& Makram-Ebeid (1979) and Ganichev \& Prettl (1997), which describe emission due to phonon assisted tunneling, were fitted to the observed electric field dependence of the emission rate. The model of Pons \& Makram-Ebeid fitted the measured emission rate more accurately than Ganichev \& Prettl. However the model of Ganichev \& Prettl has only two parameters, while the model of Pons \& Makram-Ebeid has four. Both models showed a transition in the dominant emission mechanism from a weak electron-phonon coupling below $152.5 \mathrm{~K}$ to a strong electronphonon coupling above $155 \mathrm{~K}$. After the application of a $\chi^{2}$ goodness of fit test, it was determined that the model of Pons \& Makram-Ebeid describes the data well, while that of Ganichev \& Prettl does not.

\section{PACS Keywords:}

Phonon assisted tunneling, Field enhanced emission

\section{Introduction}

Germanium has been used as a semiconductor since the 1940s but more recently has found uses in far infrared detectors [1]. Defects in semiconductors may have a significant influence on the performance of photonic devices fabricated on the semiconductor, influencing parameters such as dark current and response time. This is due to the emission and capture of charge carriers by the defect. Except for direct thermal emission, there are a number of other mechanisms that might play a role in the emission of carriers from defects. The mechanism considered here is phonon assisted tunneling. Two models are considered that describe phonon assisted tunneling, namely those described by Pons \& Makram-Ebeid [2] and Ganichev \& Prettl [3].

In the model described by Pons \& Makram-Ebeid [2], phonon assisted tunneling occurs in defects with significant electron-lattice coupling. This coupling causes the formation of a series of quasi states within the potential well of the trap, separated by an energy $\hbar \omega$, which is the energy of the associated phonons. Elastic tunneling then occurs from any of these quasi states towards the conduction band through the walls of the potential well.

The field enhanced emission rate due to phonon assisted tunneling, $e(E)$, as derived by Pons \& Makram-Ebeid [2] is then

$$
e(E)=\sum_{p} \Pi_{p} \Gamma\left(\Delta_{p}\right)\left(1-f_{1, p}\right)
$$


The function $\left(1-f_{1, p}\right)$ is the Fermi-Dirac probability of finding an empty state within the conduction band to tunnel to, $\Gamma\left(\Delta_{\mathrm{p}}\right)$ is the probability of tunnelling emission occurring for an electron in a quasi-state $\mathrm{p}$ with energy $\Delta_{\mathrm{p}}$ above the ground state, and $\Pi_{\mathrm{p}}$ is the probability of en electron being located in a quasi-state $\mathrm{p}$. A term $\mathrm{e}_{0}$ is added to this emission rate to correct for the emission rate at zero field.

The model described by Ganichev \& Prettl [3] describes the phonon assisted tunneling in terms of the tunneling time $\tau$ of an electron under the potential of the defect. This model can be applied to defects with both weak and strong electron-phonon coupling. According to Ganichev \& Prettl, the model for the field enhanced emission due to phonon assisted tunneling is:

$$
e(E)=e_{0} \exp \left(\tau_{2}^{3} e^{2} E^{2} / 3 m^{*} \hbar\right)
$$

where $\tau_{2}$ is the tunnelling time under the potential curve of an ionized impurity for a free electron with zero kinetic energy, $E$ is the electric field strength and $m^{*}$ is the effective mass of an electron in the semiconductor. This tunneling time can be related to the tunneling time $\tau_{1}$ under the potential curve for the electron bound to the impurity by

$$
\tau=\tau_{2} \pm \tau_{1}=\hbar / 2 k_{B} T
$$

The minus sign above correspond to the case for weak electron-phonon coupling and the positive sign to strong electron-phonon coupling.

Although these two models describe the same phenomenon, the functional dependence of the emission rate on the electric field predicted by these two models, differs. The model of Ganichev \& Prettl [3], predicts the natural logarithm of the emission rate of the defect to be directly proportional to the square of the field strength. The model of Pons \& Makram-Ebeid [2] does not provide such a direct relation between the emission rate of the defect and the field strength. In fact the dependence predicted by this model cannot be expressed explicitly in such a simple manner.

In this paper, we investigate the applicability of these two models to a defect introduced by inductively coupled plasma (ICP) etching in n-type Ge, with an energy level $0.31 \mathrm{eV}$ below the conduction band.

\section{Sample Preparation and Measurements}

The sample, in which the defect studied here was introduced, was a bulk-grown $n$-type Ge sample doped with $\mathrm{Sb}\left(2.5 \times 10^{15} \mathrm{~cm}^{-3}\right)$. The sample was degreased and then etched in $5 \%$ $\mathrm{H}_{2} \mathrm{O}_{2}$ for $1 \mathrm{~min}$. Ohmic contacts were formed by resistive evaporation of AuSb (0.6\%) onto the back surface of the sample, after which the sample was annealed at $350^{\circ} \mathrm{C}$ in $\mathrm{Ar}$ for 10 min to reduce its contact resistance. The sample was then etched again on the surface onto which the Schottky contacts were to be deposited using ICP (Ar) for 10 min before $\mathrm{Pd}$ contacts were deposited [4].

Field dependent Laplace DLTS [5] measurements were taken by subtracting two DLTS transients obtained with the same reverse bias but different filling pulse heights, thereby 
sampling only a narrow range of depth below the surface. Since the electric field in a Schottky diode varies with depth, it follows that by measuring defects at different depths, it is possible to measure defects under different electric fields. The magnitude of the electric field may be determined from the reverse bias voltage applied to the contact. The Laplace DLTS measurements for the $\mathrm{EP}_{0.31}$ defect were studied at temperatures ranging from $142.5 \mathrm{~K}$ to $165 \mathrm{~K}$.

The models for the phonon-assisted tunneling field enhanced emission, as described by both Pons \& Makram-Ebeid and Ganichev \& Prettl, were developed in Maple 13 [6], and best fits were obtained for each model for the defect at each temperature.

\section{Results}

The measured emission rates at each temperature were initially compared to the model of Ganichev \& Prettl. The only parameters extracted from this model are the tunneling time $\tau_{2}$ and the zero field emission $e_{0}$. From this, using eq (3), it is possible to extract the tunneling time $\tau_{1}$, which is independent of temperature. Shown in Fig. 1 is the tunneling time $\tau_{2}$ plotted vs $1000 / \mathrm{T}$.

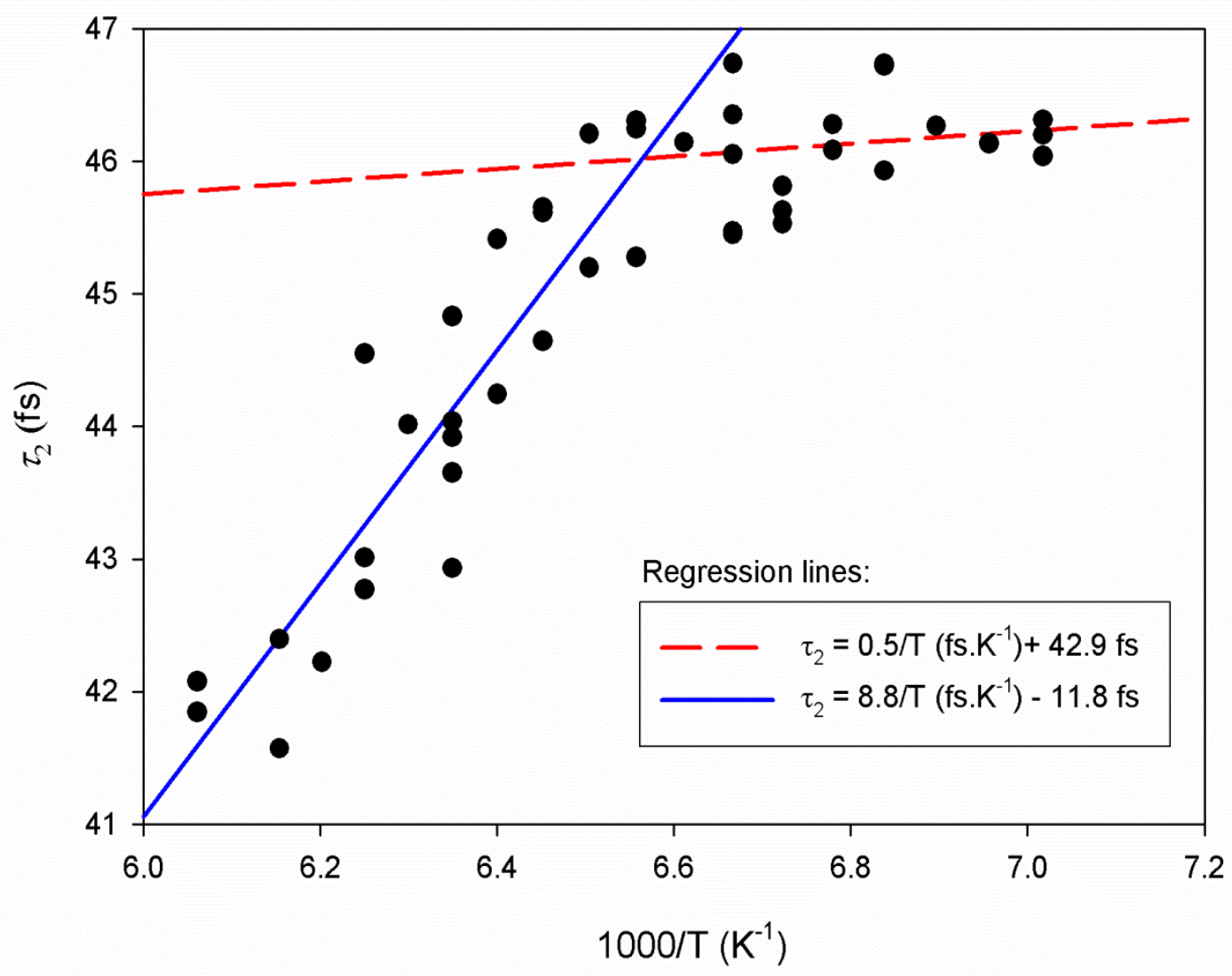

Figure 1: Tunnelling time $\tau_{2}$ vs 1000/T for the $\mathbf{E P}_{0.31}$ defect. The change in the dominant emission process can be seen. The equation of the regression lines is shown in the legend. 
Evidently a change occurs in the nature of the dominant emission process between $152 \mathrm{~K}$ and $155 \mathrm{~K}$, as is shown in Fig. 1. At lower temperatures, the tunneling time $\tau_{2}$ remains relatively constant. The defect has a positive tunnelling time $\tau_{1}$ under the potential curve of an electron bound to the impurity. This, according to Ganichev \& Prettl [3], indicates a weak electronphonon coupling. At higher temperatures, the tunneling time $\tau_{2}$ for the electron under the potential curve of the ionized impurity decreases with increasing temperature. Additionally the tunneling time $\tau_{1}$ is now negative, indicating a strong electron phonon coupling, according to [3].

The model of Pons \& Makram-Ebeid was fitted to the data, from which the Huang-Rhys factor $\mathrm{S}$, the barrier shape factor $\gamma$, the phonon energy $\hbar \omega$ and zero field emission $e_{0}$ can be extracted. During the modelling it was noticed that in many cases there was a strong dependence of the parameters on one another, and a wide range of values provided an acceptable fit. Consequently, a fixed value of $0.012 \mathrm{eV}$ was chosen for the phonon energy, as it provided a good fit over the temperature range considered. Plotted in Fig. 2 is the value of S obtained from the fitting as a function of 1000/T.

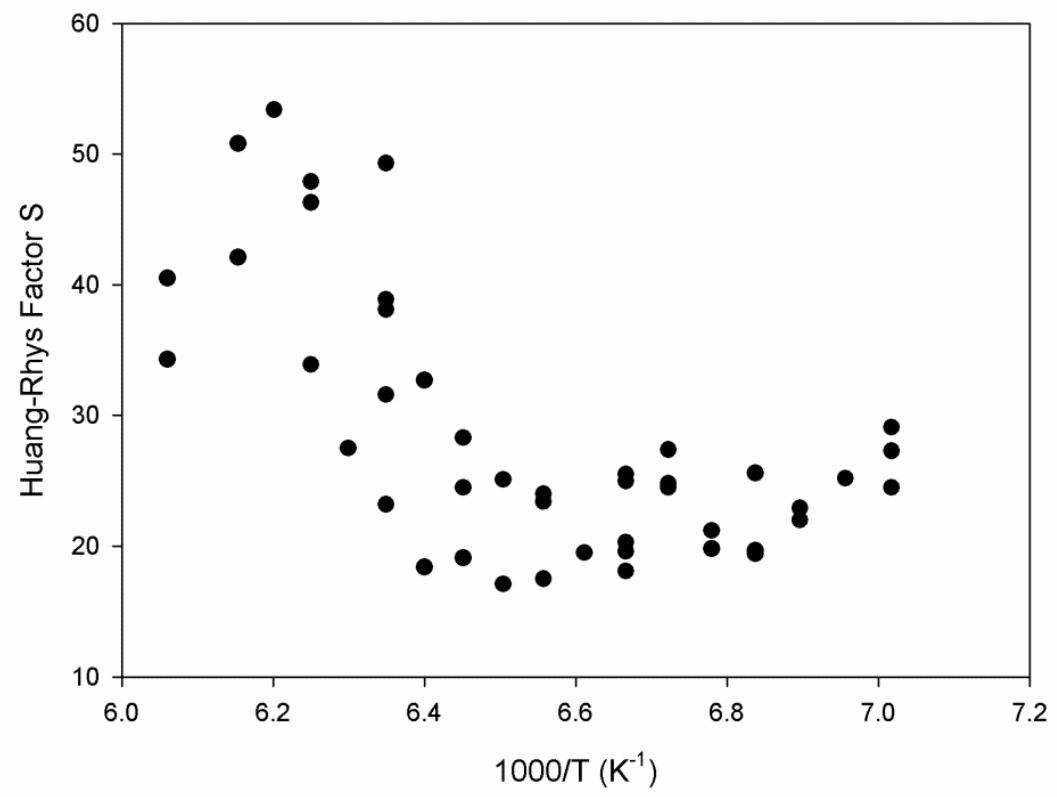

Figure 2: Huang-Rhys factor $S$ vs $1000 / T$ for the $\mathbf{E P}_{0.31}$. This illustrates the increasing trend in the value of the Huang-Rhys factor for temperatures above approximately $153 \mathrm{~K}$, indicating stronger coupling.

In Fig. 2, a transition is again evident. As was the case with the model of Ganichev \& Prettl, the transition lies in the range of $152 \mathrm{~K}$ to $155 \mathrm{~K}$. At temperatures below this transition, the Huang-Rhys factor appears to have an average value in the low 20's. At temperatures higher than this transition, the value of the Huang-Rhys factor shows a definite increasing trend. This higher range would indicate a stronger electron-phonon coupling. The results of the Pons \& Makram-Ebeid model would then be consistent with that of the Ganichev \& Prettl model which indicates a transition from a weaker electron photon coupling to strong electronphonon coupling. This transition was observed for all reasonable choices of $\hbar \omega$. 


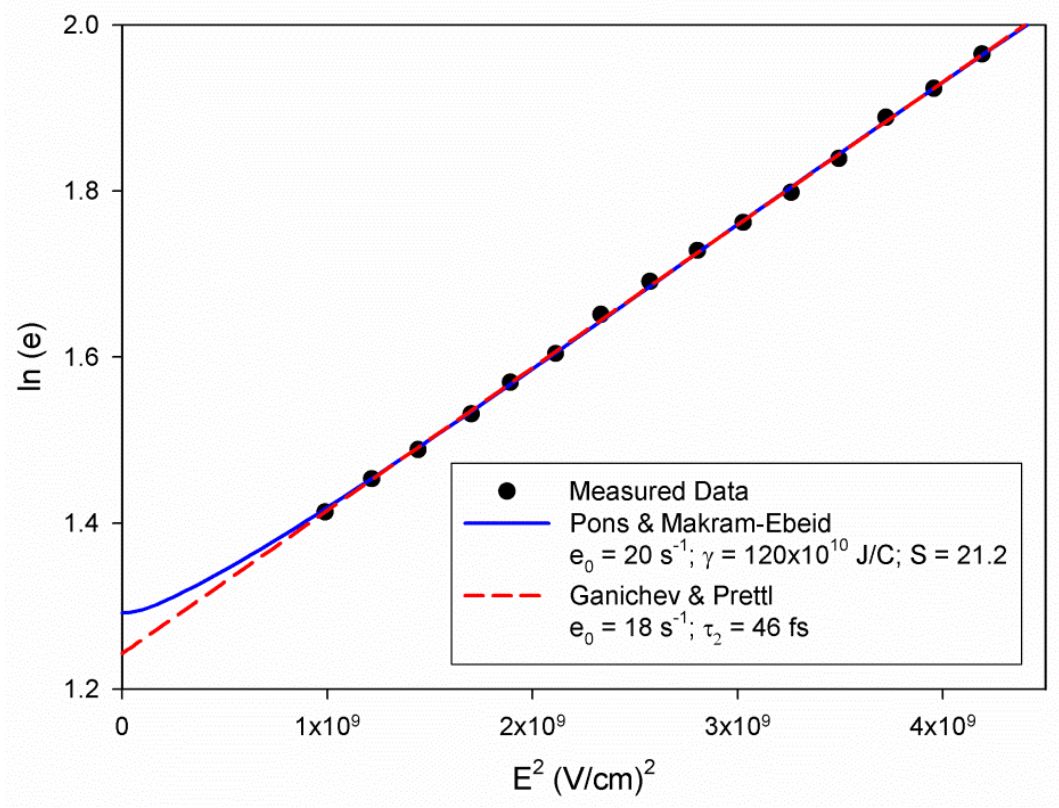

Figure 3: Plot of the ln of the emission rate vs the square of the field for nearly linear data. The model of Ganichev \& Prettl underestimates the low-field emission values.

During the modeling of the two models it was found that the Ganichev \& Prettl model does not describe the experimental curves as well as the model of Pons \& Makram-Ebeid. This becomes especially clear when considering plots of the $\ln$ of the emission rate vs the square of the field strength [Fig 3,4]. The Ganichev \& Prettl model predicts a linear relationship, however all the data sets at each temperature showed deviations from linearity which were ably predicted by the model of Pons \& Makram-Ebeid.

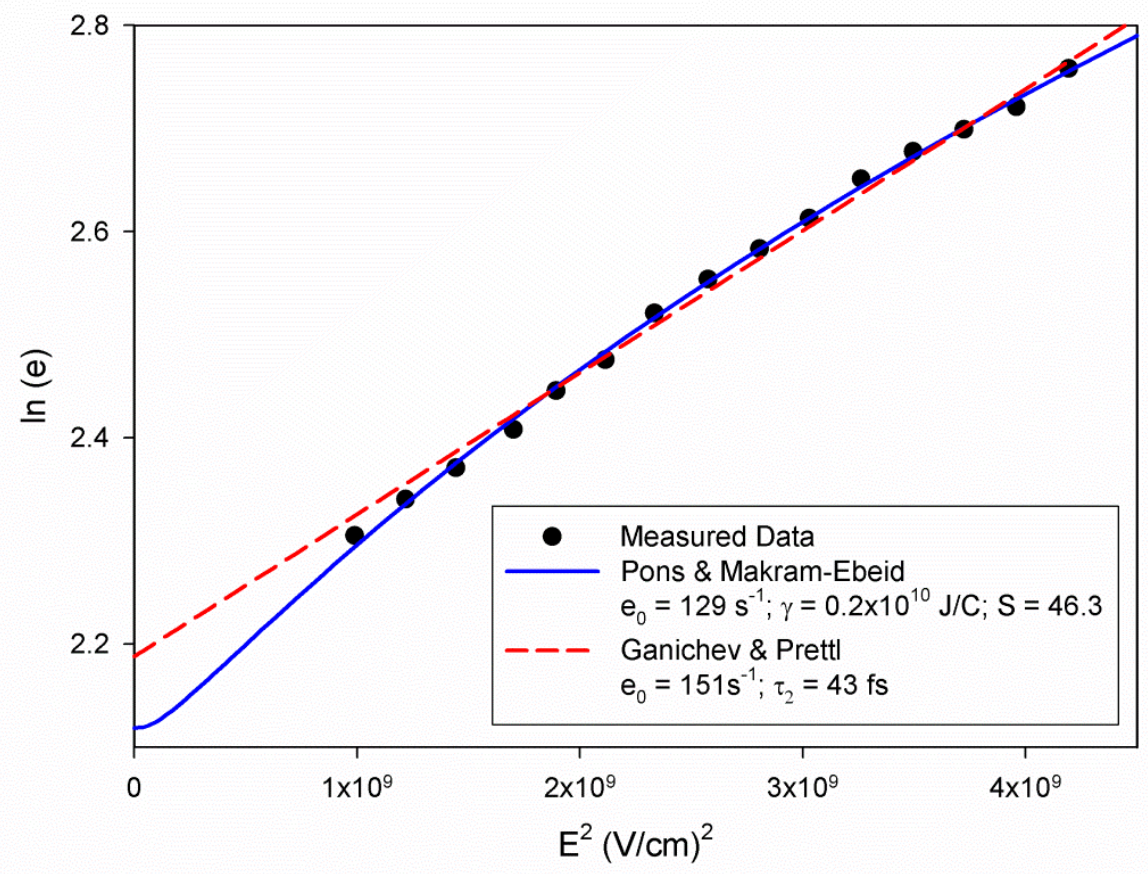

Figure 4: Plot of the In of the emission rate vs the square of the field for negatively curved data. The model of Ganichev\&Prettl over estimates the low-field emission values. 
In the low field region, the Ganichev \& Prettl model deviated significantly from the measured emission rates. When the data had a negative curvature (at temperatures above $154 \mathrm{~K}$ ), the model predicted a higher emission rate than was observed. However, the model under estimated the low-field emission rate when the data was close to linear.

From the plots of the $\ln$ of the emission rate vs the square of the field it was apparent that the data generally did not follow a linear trend. However, the model of Ganichev \& Prettl predicts a linear dependence between the square of the field and the ln of the emission rate in eq (2). The Pons \& Makram-Ebeid model on the other hand can predict the nonlinearity of the relationship between $\ln$ of emission rate and the square of the field.

It was noted that, in the model of Pons \& Makram-Ebeid, when S was kept fixed and the value of $\hbar \omega$ varied, there were specific values of in the value of $\hbar \omega$, at which the shape of the predicted field dependence changed quite drastically in the low field region. These abrupt changes arise due to the model considering discrete quasi states in the potential well.

As the value of $\hbar \omega$ changes, the number of quasi states in the well changes in discrete steps. With increasing $\hbar \omega$, this implies that the number of quasi states decreases. Just before such a decrease, the top most quasi level lies just below the top of the energy well of the trap. When an electric field is applied, the triangular barrier through which electrons must tunnel in order to be emitted is very thin and emission occurs easily, as a result the field enhancement of the emission is over estimated and the zero field emission term $e_{0}$ is underestimated.

Conversely at a phonon energy just above this transition, one quasi level is discarded from the summation, as this level will lie just above the energy well of the trap. This results in the next phonon level lying well below the top of the energy well. Tunneling from this level consequently becomes much more unlikely, as the distance through which the electron must tunnel becomes much greater. As a result the field enhanced emission is under estimated and the zero field emission term $e_{0}$ is over estimated.

Using the zero field emission rates in Arrhenius plots, the depth below the conduction band at which the defect lies was determined. This was found to be $0.312 \mathrm{eV}$ using the model of Ganichev \& Prettl and $0.315 \mathrm{eV}$ using the model of Pons \& Makram-Ebeid. The apparent capture cross sections were also determined to be $1.7 \times 10^{-13} \mathrm{~cm}^{2}$ for the Pons \& MakramEbeid model and $1.4 \times 10^{-13} \mathrm{~cm}^{2}$ for the Ganichev \& Prettl model.

To test the appropriateness of the two models, a $\chi^{2}$ suitability of model test was conducted on each model at a $99.5 \%$ confidence level. It was found that, in the case of the Pons \& Makram-Ebeid model, only five of 47 fits (i.e. 10\%) were rejected, while in the case of Ganichev \& Prettl nearly half of the fits were found to be unsuitable.

\section{Conclusion}

It was found that the model for phonon assisted tunneling as described by Pons \& MakramEbeid produces meaningful fits to observed emission data and is able to predict nonlinear trends in the data. However, the model by Pons \& Makram-Ebeid has four parameters and it is more difficult to fit to a data set. Also the discrete nature of the quasi states must be considered when fitting solutions, as these affect the form of the solution dramatically at certain transition points in the phonon energy. The model of Ganichev \& Prettl does not fit 
the emission rate as well as that of Pons \& Makram-Ebeid, however it has only two parameters and is consequently easier to fit. In the case of the $\mathrm{EP}_{0.31}$, both models provided consistent values for the ionization enthalpy of the defect at zero electric field. Both models also showed that a transition occured in the dominant emission process from a state where weak electron-phonon coupling existed to a state whether the electron-phonon coupling was strong.

\section{Acknowledgements}

This work has been made possible by financial assistance from theSouth African National Research Foundation. The Laplace DLTS software and hardware used here was received from L. Dobaczewski (Institute of Physics, Polish Academy of Science) and A. R Peaker (Centre for Electronic Materials Devices and Nanostructures, University of Manchester).

\section{References}

[1] E. E. Haller, Mater. Sci. Semicond. Proc.9 (2006) 408

[2]D. Pons, S. Makram-Ebeid, J. Phys. (Paris) 40 (1979) 1161

[3]S.D. Ganichev, W. Prettl Phys. Solid State 39 (1997) 1703

[4] F.D. Auret, S.M.M. Coelho, G. Myburg, P.J. Janse van Rensburg, W.E. Meyer_Physica B: Condensed Matter, 404(22) (2009)4376-4378

[5] L. Dobaczewski, P. Kaczor, I.D. Hawkins, A.R. Peaker, J. Appl. Phys. 76 (1994) 194

[6] Maplesoft, Waterloo, Canada 